\title{
Experimental study of breakaway system for the fast-steering secondary mirror prototype of GMT
}

\author{
Yunjong Kim, Wongi Lee, Chanhee Lee, Myung K. Cho, \\ Christoph Dribusch, et al.
}

Yunjong Kim, Wongi Lee, Chanhee Lee, Myung K. Cho, Christoph Dribusch, Sungho Lee, Sanghyuk Kim, Chang-Hee Kim, Ueejeong Jeong, Jeong-Yeol Han, Bong-Kon Moon, Chan Park, Byeong-Gon Park, Youra Jun, Jieun Ryu, Gary Poczulp, Ming Liang, II-Kwon Moon, Ho-Sang Kim, Paul Gardner, Robert Bernier, Frank Groark, Hugo Chiquito, "Experimental study of breakaway system for the fast-steering secondary mirror prototype of GMT ," Proc. SPIE 10706, Advances in Optical and Mechanical Technologies for Telescopes and Instrumentation III, 107064A (10 July 2018); doi: $10.1117 / 12.2311779$

Event: SPIE Astronomical Telescopes + Instrumentation, 2018, Austin, Texas, United States 


\title{
Experimental Study of Breakaway System for the Fast-steering Secondary Mirror Prototype of the GMT
}

\author{
Yunjong Kim*a, Wongi Lee ${ }^{\mathrm{e}}$, Chanhee Lee ${ }^{\mathrm{e}}$, Myung K. Cho ${ }^{\mathrm{b}}$, Christoph Dribusch ${ }^{\mathrm{c}}$, Sungho Lee ${ }^{\mathrm{a}}$ \\ Sanghyuk Kim ${ }^{\mathrm{a}}$, Chang-Hee Kim ${ }^{\mathrm{a}}$, Ueejeong Jeong, ${ }^{\mathrm{a}}$, Jeong-Yeol Han ${ }^{\mathrm{a}}$, Bong-Kon Moon ${ }^{\mathrm{a}}$, Chan \\ Park $^{\mathrm{a}}$, Byeong-Gon Park ${ }^{\mathrm{a}}$, Youra Jun ${ }^{\mathrm{c}}$, Jieun Ryu ${ }^{\mathrm{b}}$, Gary Poczulp ${ }^{\mathrm{b}}$, Ming Liang ${ }^{\mathrm{b}}$, Il-Kwon Moon ${ }^{\mathrm{d}}$, \\ Ho-Sang Kim ${ }^{\mathrm{e}}$, Paul Gardner ${ }^{\mathrm{f}}$, Robert Bernier ${ }^{\mathrm{f}}$, Frank Groark ${ }^{\mathrm{f}}$, Hugo Chiquito ${ }^{\mathrm{f}}$ \\ ${ }^{\mathrm{a}}$ Korea Astronomy and Space Science Institute, Daejeon 34055, Republic of Korea; \\ bNational Optical Astronomy Observatory, 950 N. Cherry Ave., Tucson, AZ 85719, USA; \\ ${ }^{c}$ University of Arizona, Tucson, AZ 85721, USA; \\ ${ }^{\mathrm{d}}$ Korea Research Institute of Standards and Science, Daejeon 34113, Republic of Korea; \\ e Institute for Advanced Engineering, Yongin-si, Gyeonggi-do 17180, Republic of Korea; \\ ${ }^{\mathrm{f}}$ GMTO Corporation, 465 N. Halstead Street, Suite 250, Pasadena, CA 91107, USA
}

\begin{abstract}
The Fast-steering Secondary Mirror (FSM) of Giant Magellan Telescope (GMT) consists of seven $1.1 \mathrm{~m}$ diameter circular segments with an effective diameter of $3.2 \mathrm{~m}$, which are conjugated 1:1 to the seven $8.4 \mathrm{~m}$ segments of the primary. Each FSM segment contains a tip-tilt capability for fast guiding to attenuate telescope wind shake and mount control jitter by adapting axial support actuators. Breakaway System (BAS) is installed for protecting FSM from seismic overload or other unknown shocks in the axial support. When an earthquake or other unknown shocks come in, the springs in the BAS should limit the force along the axial support axis not to damage the mirror. We tested a single BAS in the lab by changing the input force to the BAS in a resolution of $10 \mathrm{~N}$ and measuring the displacement of the system. In this paper, we present experimental results from changing the input force gradually. We will discuss the detailed characteristics of the BAS in this report.
\end{abstract}

Keywords: Extremely Large Telescope, Giant Magellan Telescope, Fast-steering Secondary Mirror, Breakaway System, Tip-Tilt

\section{INTRODUCTION}

The Giant Magellan Telescope (GMT) is a 25-meter ground-based optical/infrared extremely large telescope which is featured with two Gregorian secondary mirrors, an Adaptive Secondary Mirror (ASM) and a Fast-steering Secondary Mirror (FSM $)^{[1]}$. The FSM and ASM segments share a common optical prescription and a common interface with the M2 positioning system. The FSM consists of seven $1.1 \mathrm{~m}$ diameter circular segments with an effective diameter of $3.2 \mathrm{~m}$, which are conjugated 1:1 to the seven $8.4 \mathrm{~m}$ segments of the primary. Figure 1 shows a conceptual diagram of the assembled FSM at top end structure ${ }^{[2]}$. The FSM will be the first implemented secondary mirror on the GMT. It will be used in the initial engineering phase and commissioning of the telescope. It will also be used for seeing-limited observations at times when the ASM needs to be removed for maintenance or repair ${ }^{[2]}$.

FSM segments are capable of producing rapid, small tip-tilt motions to compensate for wind-induced vibration and tracking jitter by adapting axial supports ${ }^{[3]-[5]}$. This tip-tilt capability thus enhances the performance of the telescope in the seeing limited observation mode. Each segment is held in three axial supports and a central lateral support with a vacuum system for pressure compensation as shown in Figure 2(a). The axial supports represent a solid link between the mirror and mirror cell and constrain three degrees-of-freedom: tip, tilt, and piston (local z-axis). Figure 2(b) shows the detailed axial support. Each axial support consists of the following key components: 1) a breakaway system for seismic

*yjkim@kasi.re.kr; phone 8242 865-2181; fax 8242 865-2125

Advances in Optical and Mechanical Technologies for Telescopes and Instrumentation III, edited by Ramón Navarro, Roland Geyl, Proc. of SPIE Vol. 10706, 107064A · (c) 2018 SPIE CCC code: $0277-786 X / 18 / \$ 18 \cdot$ doi: $10.1117 / 12.2311779$ 
overload protection 2) a piezo actuator for tip-tilt motion, 3) two flexible tip flexures to accommodate tip-tilt motion, 4) a load cell for sensing the forces along the axis of the axial support, and 5) preload springs to keep the piezo actuator in compression.

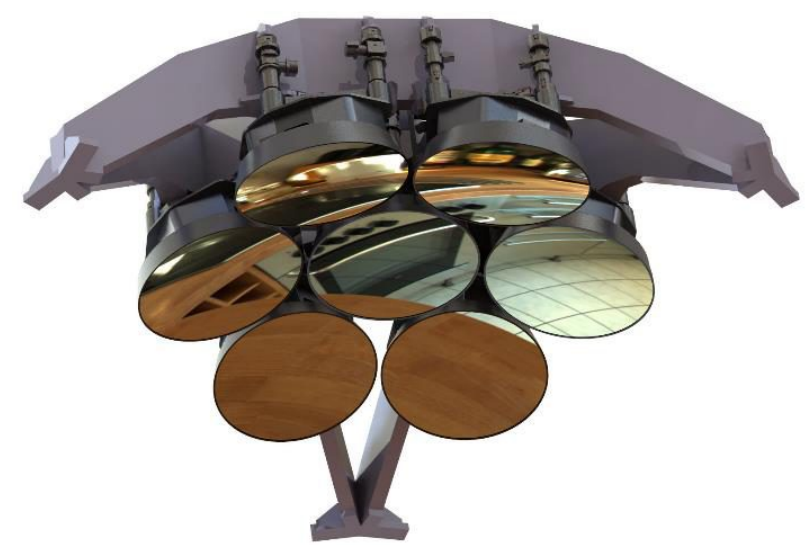

Figure 1. A conceptual diagram of the assembled FSM at top end structure for the M2 global positioner.

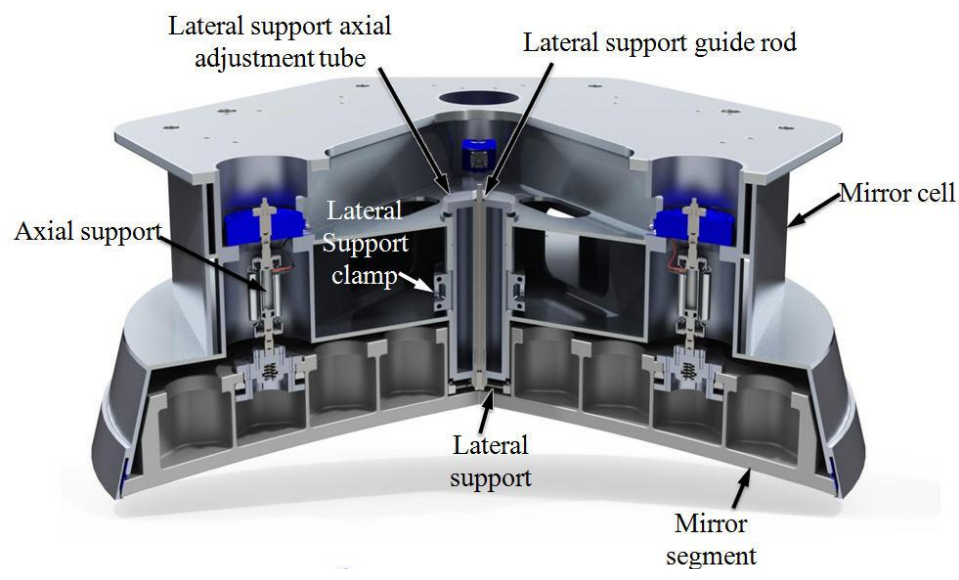

(a)

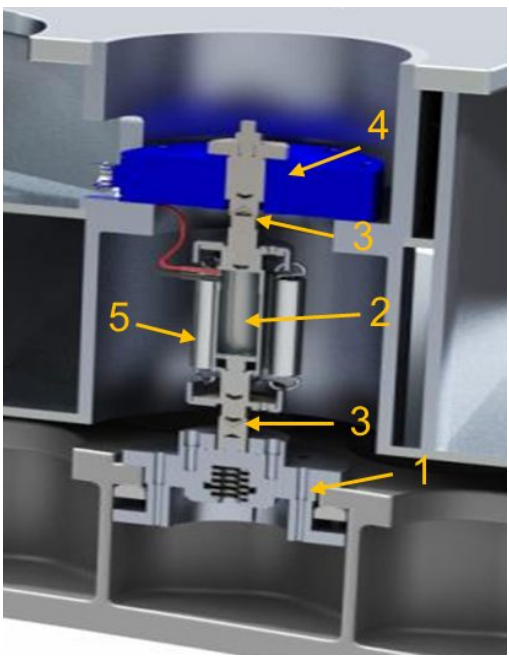

(b)

Figure 2. (a) Section view of an off-axis FSM. The components are axial support, lateral support, mirror cell and mirror segment. (b) One of the axial support. The components are 1) a breakaway system for seismic overload protection, 2) a piezo actuator for tip-tilt motion, 3) two flexible tip flexures to accommodate tip-tilt motion, 4) a load cell for sensing the forces along the axis of the axial support, and 5) preload springs to keep the piezo actuator in compression

Breakaway system(BAS) will be installed at the contact place of the back surface of the mirror as shown in Figure 2. The breakaway system is a safety device to avert any possible physical damage on the mirror against a sudden impact or seismic acceleration. When an earthquake or other unknown shocks come in, the springs in the system should limit the force along the axial support axis not to damage the mirror. The springs would disconnect the axial support and the mirror body only if an external force greater than the designed preload. The BAS design has a balancing feature with sets of springs in push and pull.

We carried out a single BAS test for Fast-steering Secondary Mirror Prototype(FSMP) in the lab, instead of making a system for FSM. We verified the systematic performance of the inner and outer spring system through the experimental 
result of BAS for FSMP. We changed the input force to the BAS in $10 \mathrm{~N}$ resolution and measuring the displacement of the system up to $1000 \mathrm{~N}$. In section 2, we briefly explain the working mechanism of the BAS and describe the setup for push and pull test. In section 3, we present the lab results and discussions of the current BAS and test setup. We conclude briefly in section 4 .

\section{EXPERIMENTAL SETUP}

\subsection{Breakaway System}

The role of a BAS is to prevent the mirror from translation movement due to an earthquake or another event. Determine a spring specification such as spring preload, spring constant, the deflection length is a key factor for successful operation of BAS. Figure 3 shows a definition of spring dimensions ${ }^{[6]}$. The free length is the spring length without any load on the spring. When a force is adapted to the spring, the variation of the spring length is the total deflection length and the difference of the free length and the total deflection length is the compressed length. The maximum preload is the breakaway force. The restoring force increases with the breakaway travel. The requirement of the preload of the spring is one to three times of gravity force of mirror ${ }^{[7]}$. The mass of the mirror is nearly $100 \mathrm{~kg}$ and there are three axial

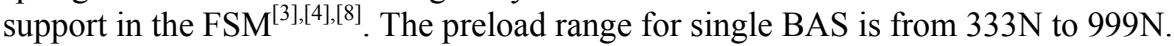

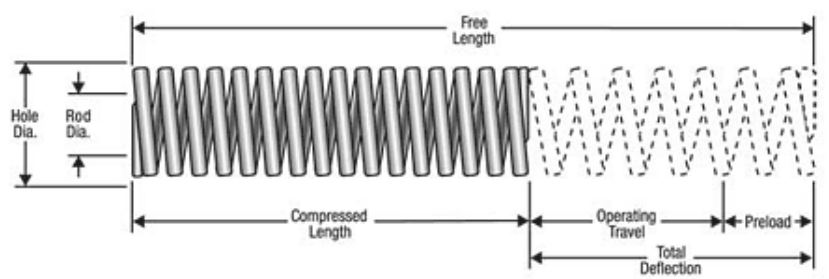

Figure 3. Definition of the spring part ${ }^{[6]}$.

Figure 4(a) shows a BAS design for the FSM and Figure 4(b) shows a section view of the BAS. It consists of upper and lower part with springs. FSM is located between lower and upper part. The spring in the upper part is to protect the FSM for downward force and the springs on the lower part is for upward force. Those springs shrink when the external force is larger than the preload of the spring to protect the mirror from unintended up and down movement. The red arrows in Figure 4(b) shows force direction on the working spring part. When the downward force, which is larger than preload of the inner spring, is coming to the FSM, the inner spring in the upper part starts to shrink. When the upward force is coming to the FSM, the outer springs on the lower part start to shrink. Therefore, the upper part and the lower part will be detached.

We revised the initial BAS design simply to make the assembly procedure easier between FSMP dummy mirror and axial support. Figure 4 shows the initial BAS design for FSM and Figure 5 shows the revised BAS design for FSMP. The red arrows in Figure 5(b) shows force direction on the working spring part. The BAS design for FSMP is slightly different from the BAS for FSM, but the role of the BAS and the operation principle is the same as in both designs. The only difference is that the position of the inner spring and outer springs are opposite sides in the revised design.

There is a one inner spring in the lower part. This spring shrinks when a force makes the mirror and the mirror cell close to each other. The entire length of the axial support is reduced in this case. The model of the inner spring is MISUMI's SWB 16-25 as shown in Figure 6(a). The designed spring constant is $196 \mathrm{~N} / \mathrm{mm}$, and the free length is $25 \mathrm{~mm}^{[9]}$. We set the compressed length of the inner spring is $22 \mathrm{~mm}$ to meet the requirement. The preload is $588 \mathrm{~N}$ calculated by $196 \mathrm{~N} / \mathrm{mm} *(25-22) \mathrm{mm}$. There are six outer springs in the upper part. The outer spring is shortened when a pulling force, which makes the mirror and the mirror cell farther away, applied to the mirror. The entire length of the BAS is extended in this case. The model of the outer spring is Century's D-1101 as shown in Figure 6(b). The spring constant is $9.81 \mathrm{~N} / \mathrm{mm}$ and the free length is $25.4 \mathrm{~mm}^{[10]}$. We set the compressed length of the inner spring as $17.4 \mathrm{~mm}$, Therefore, the preload is $470 \mathrm{~N}$ calculated by $9.81 \mathrm{~N} / \mathrm{mm}^{*}(25.4-17.4) \mathrm{mm} * 6$. Figure 7 shows assembled BAS for the FSMP. 
There are six outer springs and one inner spring. We measured compressed length of the outer spring to maintain the same preload of all the parts.

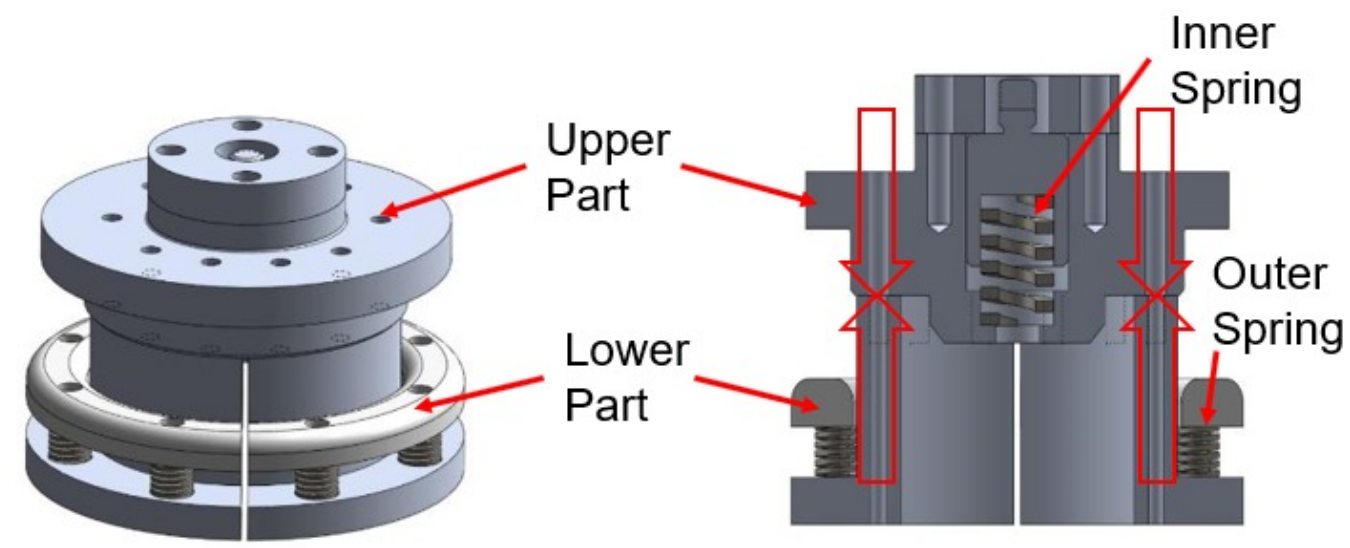

(a)

(b)

Figure 4. BAS design for FSM. It consists of upper and lower part. FSM is located between inner and outer part. (a) 3D model (b) Section view of the BAS. The red arrow means the force direction to the BAS on the working spring part.

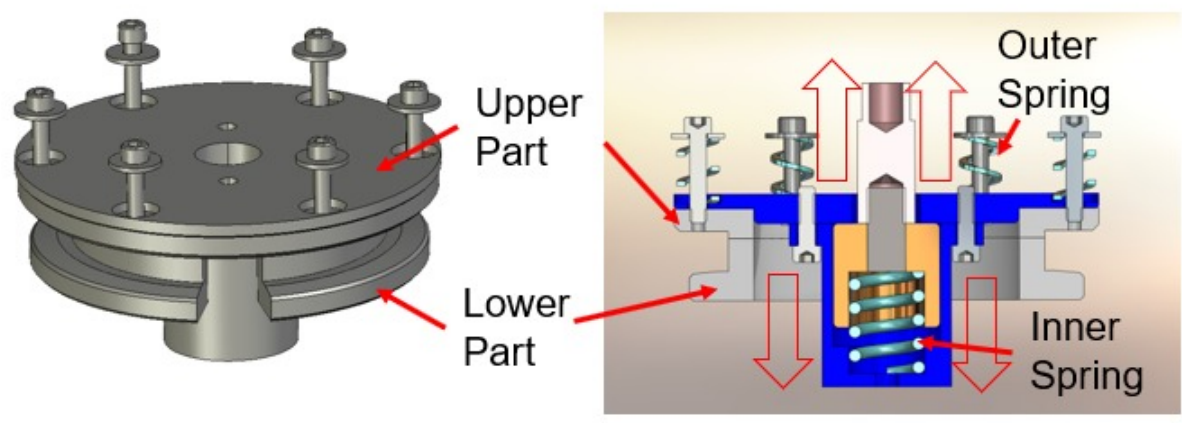

(a)

(b)

Figure 5. BAS design for FSMP. It consists of upper and lower part. (a) 3D model (b) Section view of BAS. The red arrow means the force direction to the BAS on the working spring part.

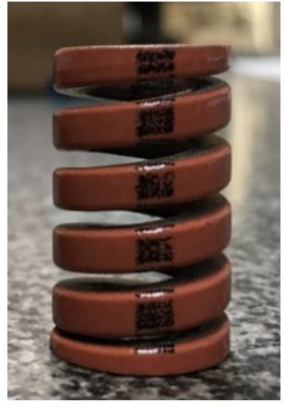

(a)

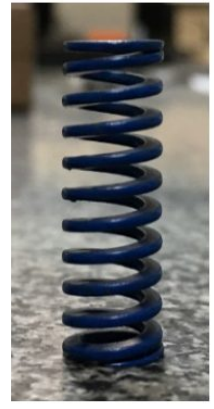

(b)

Figure 6. The springs used in the BAS for FSMP (a) MISUMI's SWB 16-25 which is used in inner spring (b) Century's D1101 which is used in outer spring 


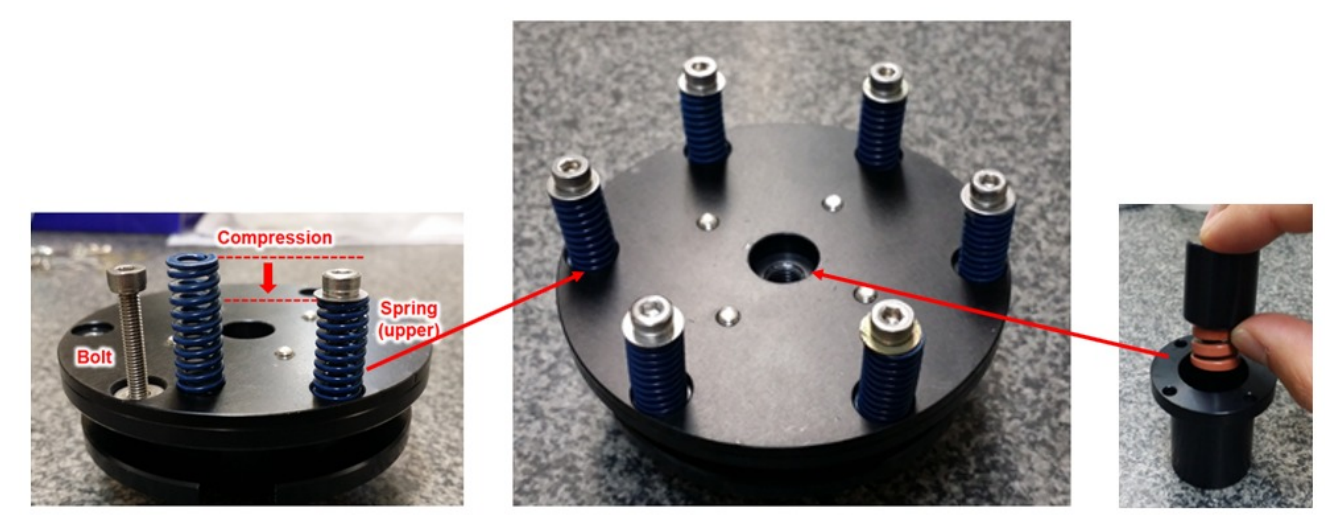

Figure 7. Assembled BAS for the FSMP. There are six outer springs and one inner spring.

\subsection{Testbed Setup}

The purpose of testing the BAS for FSMP in the lab is to verify the stiffness of the springs when a force is applied to the system. If the force is larger than a preload of the system, the BAS should be extended or reduced to protect the mirror from the force. Otherwise, the total length of the BAS will be same because the input force is less than a preload force. We can estimate whether the system works or not by measuring the length of the BAS with the input force. After we get the data at this configuration we will add axial support and see the performance of the BAS with the same condition.

Figure 8 shows a jig design for holding the BAS module to a tensile test machine. Figure 9 shows assembled BAS module for the lab test. Figure 10 shows test configuration of the BAS with the tensile test machine. We are using this test machine to input variety of forces to the BAS and record the length variations of the BAS. The cylindrical part of the upper and lower jig is attached to the upper and lower clipper of the tensile test machine. There is a load cell at the top of the upper clipper on the tensile test machine. We can record the current force through the load cell. We can also record the current position of the machine through the position sensor in the machine at the different tensile force simultaneously. Therefore, we can notice the length variation of the BAS when the force is larger than a preload of the springs.

We are considering two types of the input force. One is a gradual increase the force by $10 \mathrm{~N}$ tension from $0 \mathrm{~N}$ to $1000 \mathrm{~N}$. From this measurement we could check the mechanical properties of the BAS under experimental condition. The other is an impulse or sinusoidal force to simulate an earthquake or other unknown shocks. This condition is similar to an unexpected case in real operation. However, we don't have a facility to check this condition. Instead of doing the experiment we will perform a simulation to examine the BAS under impulse and sinusoidal force.

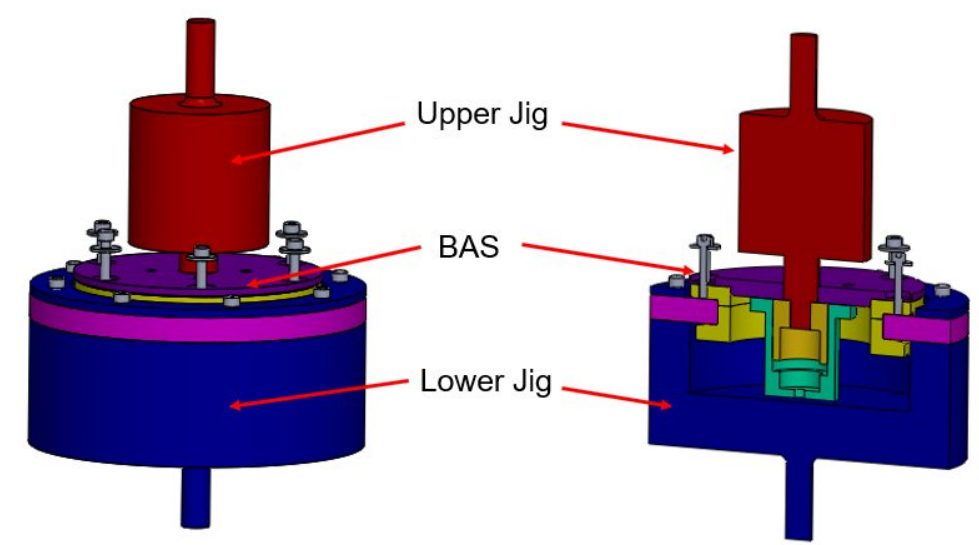

Figure 8. A jig design for holding the BAS to tensile test machine. 


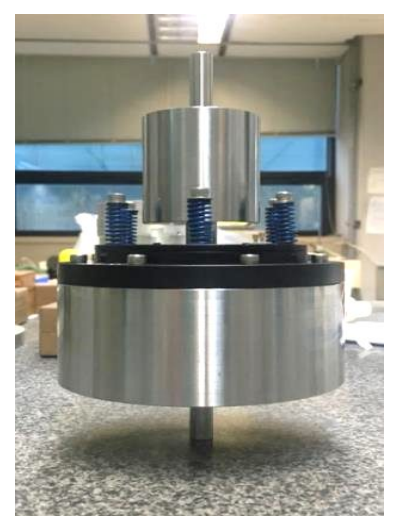

Figure 9. The assembled BAS module with the jig for lab test.

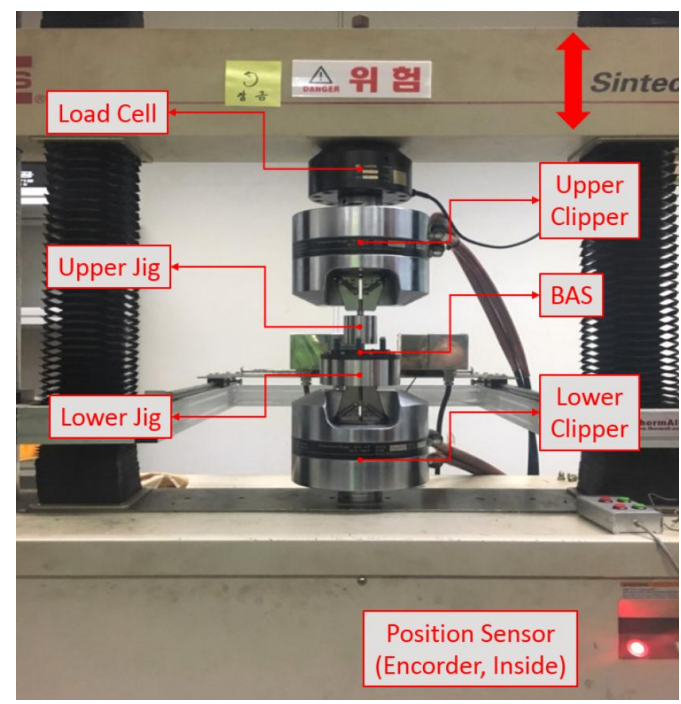

Figure 10. The assembled BAS module on the tensile test machine.

\section{RESULTS}

We carried out a tensile test in the lab to verify the performance of the BAS in the various input force. We got displacement data at push and pull input force. We changed the input force gradually to the BAS in $10 \mathrm{~N}$ resolution. Figure 11 shows displacement variation at the pushed force and Figure 12 shows displacement variation at the pulled force with five different measurements. Mea.1 to Mea.5 in the legend box represents measurement data set of each case. Average of five data set also included in these figures. For the pushed force case, the measurement condition was very stable, therefore the deviation of the data set is less than $10 \mu \mathrm{m}$, there is one inflection point and all the data look overlap. For pulled force case, the displacement deviation is less than $600 \mu \mathrm{m}$, each data set is distributed more widely compared to compression case and there are two inflection points.

We can determine the preload from these figures where the displacement change dramatically. The measured preload of the inner spring, which is for compression, is $718 \mathrm{~N}$ which is an averaged value of five different cases. The position of the preload indicated in Figure 11. The measured preload of outer spring, which is for tension, starts to varying at $370 \mathrm{~N}$. The displacement sharply increased at $604 \mathrm{~N}$. Table 1 shows designed and measured preload at inner and outer springs. The maximum difference of the preload for tension is $110 \mathrm{~N}$. The designed preload for inner spring was $588 \mathrm{~N}$ and outer spring was $470 \mathrm{~N}$. The measured preload is higher than the designed value in both inner and outer springs. 
We calculated spring constant of the inner and outer springs in the BAS to verify the difference of the designed and measured preload. When the input force is larger than preload, BAS is connected to the spring only. Therefore, we can measure the spring constant of the BAS at this force range. Table 2 shows designed and measured spring constant of the inner and outer springs.

We can estimate the deflection length of the inner and outer spring from the measured preload and spring constant. The designed deflection length could be calculated from preload of the spring divided by spring constant. The designed deflection length for inner spring is $3 \mathrm{~mm}$ and outer spring is $8 \mathrm{~mm}$. We could not measure this length directly, instead, we measure free length of the spring and compressed length of the spring. From these data, we can calculate the actual deflection length of those springs.

The free length of the spring measured before assembled those springs to the BAS module without any input force. Table 3 shows free length and compressed length of the spring of designed and measured value. The difference of free length and compress length is the actual deflection length of those springs. The deflection length of the inner spring is $2.5 \mathrm{~mm}$. The measured value is like the estimated length. All the free length of the outer spring is not the same. The average free length of the outer spring is $26.5 \mathrm{~mm}$, therefore the deflection length for the experiment will be like $8.5 \mathrm{~mm}$. The designed deflection length of outer spring is smaller than the length of the experiment.

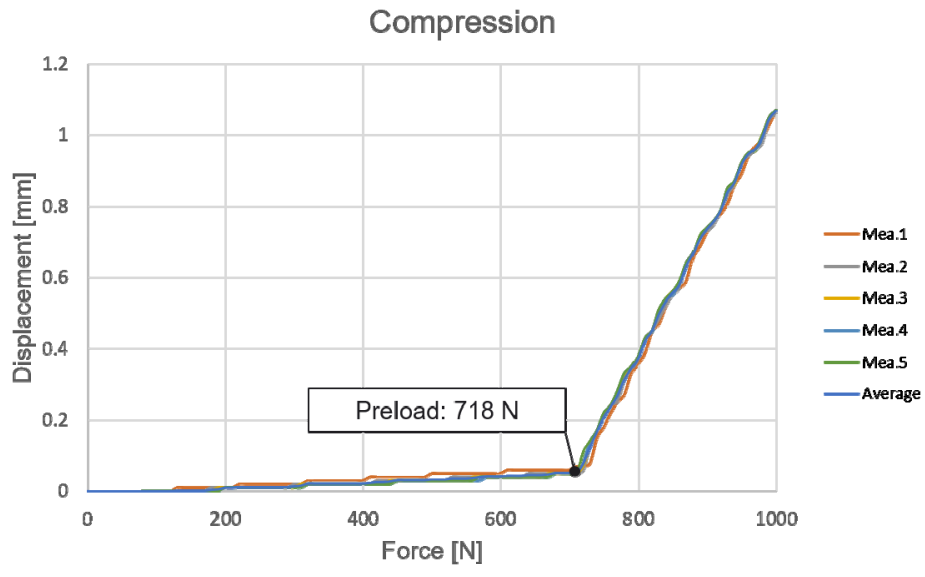

Figure 11. Displacement variation curve with compressed force.

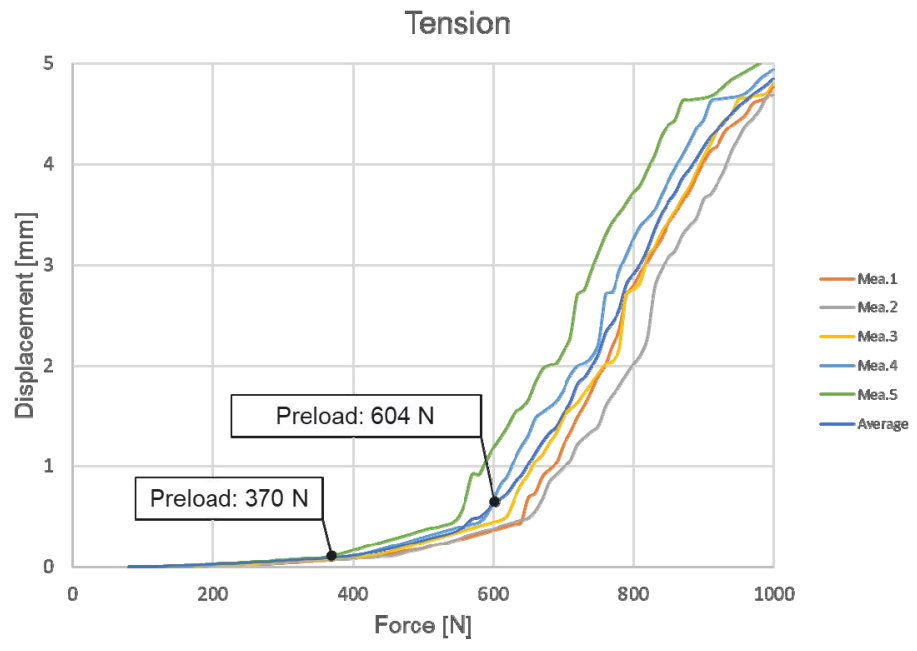

Figure 12. Displacement variation curve with pulled force. 
Table 1. Designed and measured preload of inner and outer spring.

\begin{tabular}{c|ccc}
\hline Unit: N & Designed Preload & Measured Preload \\
\hline \multirow{3}{*}{ Inner Spring } & & 730 & \\
& 588 & 720 & 718 \\
& & 720 & \\
& & 710 & \\
& & 710 & 604 \\
\hline \multirow{2}{*}{ Outer Spring } & & 640 & \\
& 470 & 650 & \\
& & 610 & \\
& & 580 & \\
& & 540 & \\
\hline
\end{tabular}

Table 2. Designed and measured spring constant.

\begin{tabular}{c|cc}
\hline Unit: N/mm & Designed Constant & Measured Constant \\
\hline Inner Spring & 196 & 284.5 \\
Outer Spring & 59 & 75.8 \\
\hline
\end{tabular}

Table 3. Free and compressed length of inner and outer spring.

\begin{tabular}{ll|cccc}
\hline \multirow{2}{*}{ Unit: $\mathrm{mm}$} & \multicolumn{2}{c}{ Designed Length } & \multicolumn{2}{c}{ Measured Length } \\
& & Free & Compressed & Free & Compressed \\
\hline \multirow{2}{*}{ Inner Spring } & 25 & 22 & 24.5 & 22 \\
& $\# 1$ & 25.4 & 17.4 & 26.3 & 18 \\
& $\# 2$ & 25.4 & 17.4 & 26.4 & 18 \\
\multirow{2}{*}{ Outer } & $\# 3$ & 25.4 & 17.4 & 26.4 & 18 \\
& $\# 4$ & 25.4 & 17.4 & 26.6 & 18 \\
& $\# 5$ & 25.4 & 17.4 & 26.6 & 18 \\
& $\# 6$ & 25.4 & 17.4 & 26.6 & 18 \\
\hline
\end{tabular}

From the experimental result, the measured preload is not the same as the designed preload. First, the measured spring constant is larger than the designed spring constant as shown in Table 2. If the deflection length were the same as designed length, the preload for the experiment will be always larger than designed preload. Second, the measured free length of the spring was not the same as the design specification as shown in Table 3 . The free length of the inner spring is $0.5 \mathrm{~mm}$ shorter than designed spring. The free length of the outer spring is approximately $1.1 \mathrm{~mm}$ longer than designed spring. Also, the designed compressed length of the outer was $17.4 \mathrm{~mm}$, but the measured compressed length of the spring was $18 \mathrm{~mm}$. Because the designed compressed value for previous design level was $18 \mathrm{~mm}$. We used previously designed equipment to compress the springs. Therefore, the deflection length of the inner spring is $0.5 \mathrm{~mm}$ shorter than the designed one and the length for outer spring is $0.5 \mathrm{~mm}$ longer than the designed value. It should be affected to the 
different preload of the BAS. Moreover, all the free length of the outer spring was not identical. The force distribution to the outer part will not be uniform. This will be a possible error source of the gradual displacement variation around preload.

\section{CONCLUSION}

We performed BAS test for FSMP with varying the pushed and pulled input force. The main purpose of this experiment is testing the working condition of BAS. We measured the displacement of the BAS at each force. From the displacement and force relation, we can estimate preload, spring constant and deflection length of the current setup to study the characteristics of the system. The intended preload for inner spring is $588 \mathrm{~N}$ and outer spring is $470 \mathrm{~N}$. The measured preload for inner spring is $718 \mathrm{~N}$ and outer spring is $604 \mathrm{~N}$. The main error source is the specification of those spring is not identical between factory catalog and actual measurement.

However, we can clearly see the BAS is working well when the input force is larger than preload. Because the displacement curve changes dramatically at preload point of experimental condition. The system should be detached when the input force is larger than preload because of the inner or outer spring shrinks. Therefore, the total length of the BAS was shrink or extend. Otherwise, the total length of the BAS would be same because the input force is less than a preload force. The actual length of BAS changed sub-mm level in this force range. We can estimate the working performance of the BAS for FSM through the experimental result of BAS for FSMP. We convinced the BAS for FSM will working well when the force is larger than $700 \mathrm{~N}$ to single axial support.

We are preparing next experiment, which will be resolved the above errors. We will check the spring specification before we put the BAS to the tensile test machine. The spring length for outer one should be identical. After that, we can clearly adjust the test result to design BAS for FSM. We are also preparing a simulation when impulse force and periodic force is coming to the breakaway system to represent an earthquake motion. In the detailed design phase, a full-scale prototype of the mirror cell and an aluminum dummy mirror will be fabricated and tested to check the feasibility and repeatability of the assembly and disassembly procedure.

\section{REFERENCES}

[1] Bernstein, R. A., McCarthy, P. J., Raybould, K., Bigelow, B. C., Bouchez, A. H., Filgueira, J. M., Jacoby, G., Johns, M., Sawyer, D., Shectman, S. and Sheehan, M., "Overview and status of the Giant Magellan Telescope project," Proc. SPIE 9145, 91451C (2014).

[2] GMTO Corp., "GMT System Level Preliminary Design Review," GMT-SE-RVW-00410 (2014)

[3] Cho, M. K., Corredor, A., Dribusch, C., Park, K., Kim, Y.-S. and Moon, I.-K., "Design and development of a fast-steering secondary mirror for the Giant Magellan Telescope,” Proc. SPIE 8125, 812505 (2011)

[4] Cho, M. K., Corredor, A., Dribusch, C., Park, W. H., Muller, G., Johns, M., Hull, C., Kern, J. and Kim Y.-S., "Development of GMT fast steering secondary mirror assembly," Proc. SPIE 9145, 91451M (2014)

[5] Lee, S., Cho, M., Park, C., Ham, J.-Y., Jeong, U., Yoon, Y.-N., Song, J. H., Park, B.-G., Dribusch, C., Park, W. H., Jun, Y., Yang, H.-S., Moon, I.-K., Oh, C. J., Kim, H.-S., Lee, K.-D., Bernier, R., Alongi, C., Rakich, A., Gardner, P., Dettmann, L. and Rosenthal, W., "Development of the fast steering secondary mirror assembly of GMT," Proc. SPIE 9912, 991241 (2016).

[6] Century Spring Corp, "Die Springs," http://www.centuryspring.com/die

[7] Kim, Y.-S., Kim, J., Song, J. H., Lee, K.-D., Kim, H.-S., Lee, C.-H., Lee, W., Choi, J. M. and Yoo, Y.-D., "GMT FSM Prototype Tip-tilt assembly procedure and test result," KASI Technical Note 15-007-140 (2015)

[8] GMTO Corp., "Fast Steering Mirror Requirements," GMT-TEL-REQ-00508 (2014)

[9] MISUMI, “Coil Springs -SWB-," https://us.misumi-ec.com/vona2/detail/110100185940/?KWSearch

[10] Century Spring Corp, "Die Springs D-1101," http://www.centuryspring.com/die-spring-d-1101.html 